ionic inhomogeneities, the detection of back-scatter echoes indicates that the scale of the fine structure in tho active region is a few metres rather than the hundreds of metres usually attributed to the $E$-region.

Grants and personnel (P.A.F.) from the Defenso Research Board of Canada have made this work possible. The radar equipment was made available through the co-operation of the Geophysical Research Directorate, Air Force Cambridge Research Center, Cambridge, Mass.

P. A. FORSYTH

F. E. VAWTER

Physics Department,

University of Saskatchewan,

Saskatoon.

Aug. 20.

${ }^{1}$ Bailey, D. K., Bateman, R., Berkncr, I. V., Booker, H. G., Montgomery, G. F., Purcell, E. M., Salisbury, W. W., and Wiesner J. 3., Phys. Rev., 88, 141 (1952)

\section{A New Method of Light Microscopy}

Usivg annular apertures made in a thin layer of candle soot on a microscope objective lens and corresponding apertures in the condenser, an ordinary microscope can be made very suitable for the study of unstained specimens. There are two different procedures.

To make higher refracting objects appear dark against a light background, it is necessary to find the objective lens surface nearest to the image of the condenser aperture and soot it by short contacts with the flame, until the transparency has diminished by one-half. On a watchmaker's lathe, using a sharp tip of some soft material, a narrow ring-shaped area is freed from soot, the diameter of the ring being greater than half the exit pupil of the objective, that is, at least twice as wide as the phase ring in conventional phase-contrast objectives. When compared with the latter, there are freedom from halo effects, increased resolving power and a pleasing gradation for objects having different refractivity.

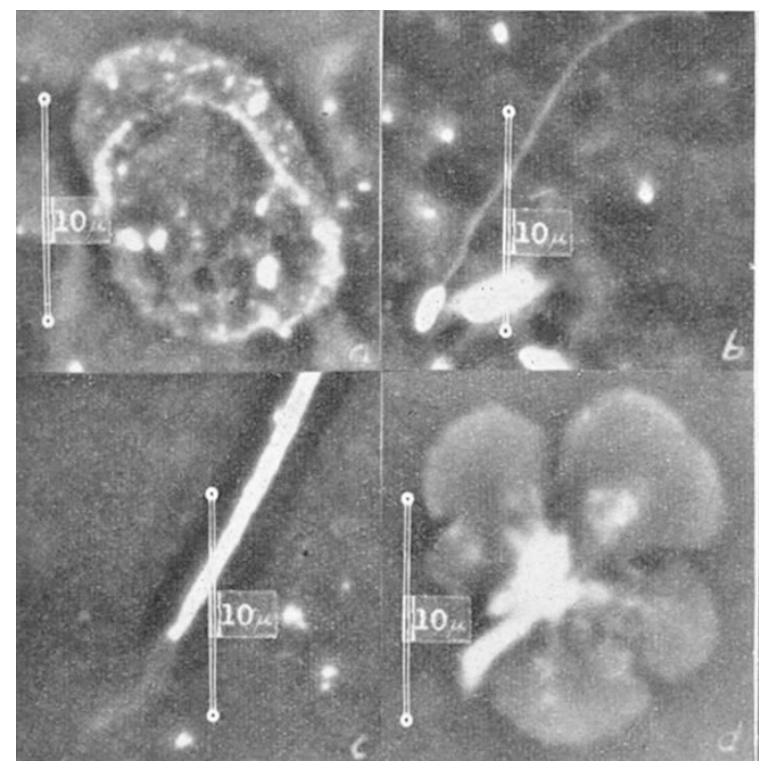

Images of greater contrast and with light values similar to those to which we are accustomed in the macroscopic world are achieved if a partly reversed procedure is followed. The sooting is continued until the transparency has decreased to 8-9 per cent, after which the soot is wiped off, except for an area covering the image of the condenser ring aperture. As a result, the background of the microscope image appears in an agreeable sepia tone, often giving the illusion of sky-illuminated objects lying on the sand floor of a shallow sea. Many features which are generally invisible with the normal use of the microscope are rendered visible; for example, influenza virus on red cell ghosts, bacterial flagella, sperm tail pencil, and delicate membranous margins in living thrombocytes (see photographs). Apparently the method can be adapted to ultra-violet microscopy and mirror optics as well.

The theoretical explanation of what occurs in each of the alternative procedures may not be an easy one, since the phase effects in microscopical observation are still incompletely understood ${ }^{1}$. The arrangements described above are the result of some hundreds of trials, in which the conditions were varied one at a time.

Institute of Physiology,

\section{A. WUSKA}

University of Helsinki.

$$
\text { Nov. } 17 .
$$

${ }^{1}$ Sec Proceedings of the London Conference on Optical Instruments, 131 (Chapman and Hall, 1951).

\section{Photoelectric Control of the Compensator in Electrophoresis}

VARIOUS types of compensator ${ }^{1-3}$ are used in electrophoresis to bring the initial boundaries into view, or to bring a particular boundary into the correct position for isolation of a sample. Manual control of the compensator is quite convenient in these cases. However, it is sometimes necessary to keep a boundary stationary during a long run, either

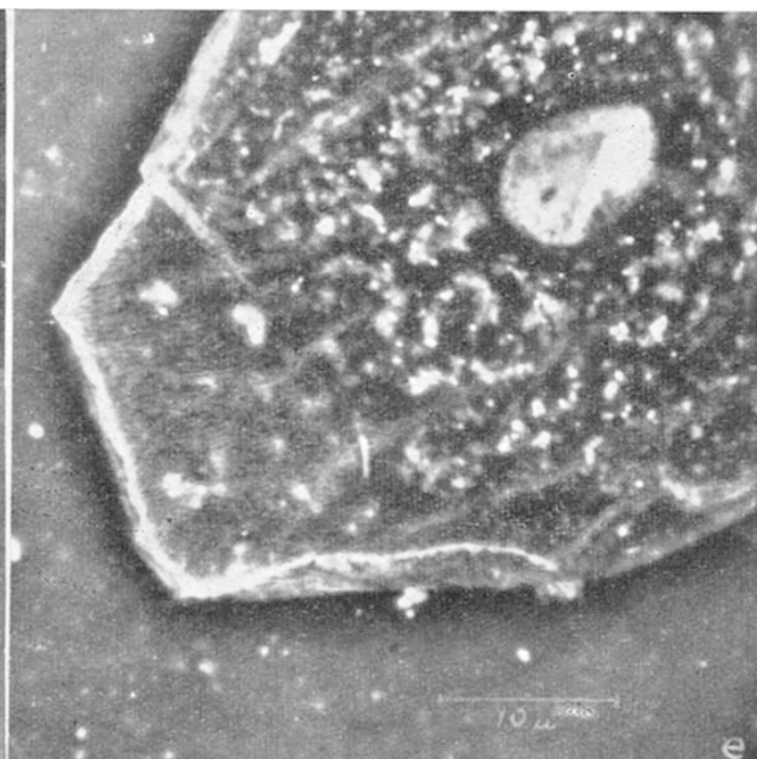

(a) Fowl erythrocyte membrane with adsorbed influenza $B$ virus ; (b) an unknown oral bacterium having a long flagellum; (c) tail of a human spermatozoon; $(d)$ a group of living human thrombocytes with spreading membranous margins; $(e)$ a cell from the gingival epithelium 